\title{
Mining of non-teleost fish transcriptomes and genomes uncovers the evolutionary history of immunoglobulin light chains
}

\author{
S. Guselnikov ${ }^{1,2 *}$, K. Baranov¹, N. Chikaev¹, A. Najakshin¹, S. Kulemzin¹, \\ A. Makunin ${ }^{1}$, V. Trifonov ${ }^{1,2}$, A. Taranin ${ }^{1,2}$ \\ ${ }^{1}$ Institute of Molecular and Cellular Biology SB RAS, Novosibirsk, Russia \\ ${ }^{2}$ Novosibirsk State University, Novosibirsk, Russia \\ *e-mail:sguselnikov@mcb.nsc.ru
}

Key words: IgL isotype, CDR length, Acipenseriformes, Polyperiformes, Holostei, Teleostei, jawed vertebrates, phylogenetic analysis

Motivation and Aim: Subdivision of mammalian immunoglobulin light (IgL) chains into kappa and lambda types has for long time remained obscure from both the functional and evolutionary perspective. The aim of this study was to fill the important gaps in the evolutionary history of light chains by examining the structure and diversity of $\operatorname{IgL}$ genes in non-teleost ray-finned fish.

Methods and Algorithms: Standard experimental (RACE PCR cDNA cloning, Sanger sequencing) and bioinformatic (BLASTN, TBLASTN, BLASTP, MUSCLE sequence alignment, NJ/ME/ML phylogenetic analysis with bootstraps) methods were used.

Results: We performed bioinformatic analysis of recent transcriptomic and/or genomic resources for four Acipenseriformes species: sterlet (Acipenser ruthenus), Siberian sturgeon (Acipenser baerii), Chinese sturgeon (Acipenser sinensis), and American paddlefish (Polyodon spathula). The results obtained were used for the detailed experimental characterization of the $\operatorname{IgL}$ genes in sterlet. This species was shown to possess three loci of genes for IgL kappa-like chains, a single Ig lambda-like VJC cluster as well as one sigma-like $\mathrm{V}$ and one sigma-like $\mathrm{C}$ gene. The data obtained on Acipenseriformes were extended by the bioinformatic identification of $\mathrm{IgL}$ genes in a holostean spotted gar (Lepisosteus oculatus) and in two polypterid species, saddled bichir (Polypterus endlicheri) and ropefish (Erpetoichthys calabaricus). The inclusion of IgL sequences from non-teleost ray-finned fish into phylogenetic analysis for the first time showed subdivision of IgL chains into five ancient types. The teleostean IgL "lambda" chains turned out to be a kappa and lambda chain paralog that emerged before the radiation of ray-finned fish but have been lost in Acipenseriformes. We designate this type lambda-2. Each vertebrate lineage has its own combination of IgL types (from one to four) with the kappa and lambda chains being the most broadly spread. Sequence comparisons showed that, in contrast to sigma, sigma-2 and lambda-2 chains, the kappa and lambda are highly variable in the length of either CDR1 (kappa) or both CDR1 and CDR2 (lambda).

Conclusion: The IgL chains are subdivided into five ancient isotypes differentially evolved in various lineages of jawed vertebrates. High variability of the CDR length in the kappa and lambda chains may be responsible for their preferential evolutionary retention by providing more flexibility in association with $\mathrm{IgH} \mathrm{V}$ domains.

Acknowledgements: Supported by the RFBR grant No. 17-04-01519 (cDNA cloning) and by the RSF grant No. 18-44-04007 (Acipenseriformes genomes and transcriptomes analysis). 